\title{
Relação de pareceristas ad hoc (2019-2020)
}

Educação e Pesquisa agradece aos especialistas abaixo relacionados, que colaboraram com a revista no período de out. 2019 a out. 2020.

Acacia Zeneida Kuenzer

Ademilson de Sousa Soares

Adenize Costa Acioli

Adir Casaro Nascimento

Adriana Almeida Sales de Melo

Adriana Backx Noronha Viana

Adriana Dragone Silveira

Adriana Garcia Gonçalves

Adriana Maria Paulo da Silva

Adriana Pugliese Netto Lamas

Adriana Sales de Melo

Adriana Varani

Adriano de Oliveira

Adriano Vargas Freitas

Airton Carrião Machado

Alan Augusto Moraes Ribeiro

Alan Silvio Ribeiro Carneiro

Alberto Moreno-Doña

Alda Junqueira Marin

Aldecilene Cerqueira Barreto

Alessandra Dias Mendes

Alessandra Frota Martinez de Schueler

Alessandra Mendes

Alessandro Roberto Oliveira

Alexander Armando Cordoves Santiesteban

Alexandre Barbosa Pereira

Alexandre Filordi de Carvalho

Alexandre Pianelli Godoy

Alexandre Ramos de Azevedo

Alexandre Saul

Alexandre Toaldo Bello

Alexandro Braga Vieira

Alexsandro da Silva

Alexsandro do Nascimento Santos

Alice Happ Botler

Alice Miriam Happ Botler

Alice Pereira Xavier Lage

Aline Lemos Pizzio

Aline Silva Dejosi Nery
Allan Moreira Xavier

Alvaro Chrispino

Amélia Cristina Abreu Artes

Amélia Cristina Abreu Artes

Amurabi Oliveira

Ana Beatriz Gomes Pimenta de Carvalho

Ana Cristina Cypriano Pereira

Ana Cristina Limongi Franca

Ana Cristina Rocha Gonçalves

Ana Cristina Troncoso

Ana Elisa Spaolonzi Queiroz Assis

Ana Isabel Mendoza Mardones

Ana Karina Amorim Checchia

Ana Laura Godinho Lima

Ana Lúcia Cunha Duarte

Ana Luísa Petersen Cogo

Ana Luiza Jesus da Costa

Ana Lydia Vasco de Albuquerque Peixoto

Ana Maria de Albuquerque Moreira

Ana Paula Caetano

Ana Paula de Oliveira Corti

Ana Paula Martinez Duboc

Ana Paula Zerbato

Ana Vergara

Ana Zimmermann

Andre Luis la Salvia

André Luis Tato Luciano Santos

André Luiz Monezi Andrade

Andreia da Silva Quintanilha Sousa

Andréia Jaqueline Devalle Rech

Andreia Lisboa Sousa Johnson

Anete Abramowicz

Ângela Francine Fuza

Angela Maria Cristina Uchoa Abreu Branco

Angela Maria Martins

Angela Maria Scalabrin Coutinho

Angelica Araujo de Melo Maia

Angelina Peralva

Aniela Improta França
Anna Augusta Sampaio de Oliveira

Antonia Almeida Silva

Antonia Terra de Calazans Fernandes

Antonio Carlos Rodrigues de Amorin

Antonio Hilario Aguilera Urquiza

Antonio João Azambuja

Antonio Sergio Abrahão Monteiro Bastos

Antonio Simplicio de Almeida Neto

Arthur Klik de Lima

Bárbara Galleli

Barbara Lutaif Bianchini

Belmira Amélia Oliveira Barros Bueno

Bruna Morelo

Bruno Cardoso de Menezes Bahia

Caio Veloso

Caio Veloso

Camila Alves Fior

Camila Ferreira da Silva

Candido Alberto da Costa Gomes

Carla Biancha Angelucci

Carlos Alberto de Oliveira Magalhães Júnior

Carlos Alberto Lopes de Sousa

Carlos Estêvão

Carlos Lopes

Carlos Monge

Carlos Pérez

Carlos Roberto da Silva Machado

Carlos Roberto Jamil Cury

Carmem Craidy

Carmem Luci da Costa Silva

Carmen Mazepa Ballão

Carmen Sylvia Vidigal Moraes

Carola Sepulveda Vasquez

Carolina Bessa Ferreira de Oliveira

Carolina de Roig Catini

Cássia Geciauskas Sofiato

Catarina Almeida Tomas

Catarina lanni Segatto

Célia Cristina de Figueiredo Cassiano 
Célia dos Prazeres Ribeiro

Célia Regina Batista Serrão

Célia Regina Rossi

Célia Regina Serrão

Celso Garcia de Araújo Ramalho

Cesar Adolfo Zamberlan

César Augusto Minto

Christiane Coutheux Trindade

Cibele Krause-Lemke

Cibele Noronha de Carvalho

Cintya Regina Ribeiro

Clarice Monteiro Escott

Cláudia Dias Prioste

Cláudia Lemos Vovio

Cláudia Maria Ribeiro

Claudia Panizzolo

Cláudio Márcio Magalhães

Cláudio Marques da Silva Neto

Cláudio Marques Martins Nogueira

Claudio Pinto Nunes

Claudio Roberto Baptista

Clecio dos Santos Bunzen Júnior

Conceição Firmina Seixas Silva

Cristiane Maria Cornelia Gottschalk

Cristiane Maria Galdino de Almeida

Cristiano Alberto Muniz

Cristiano Alberto Muniz

Cristina Broglia Feitosa De Lacerda

Cristina Ferreira de Assis

Cristovam da Silva Alves

Cynthia Greive Veiga

Cynthia Paes de Carvalho

Daielly Melina Nassif Mantova

Dalila Andrade Oliveira

Dalila Andrade Oliveira

Dandara Baçã de Jesus Lima

Daniel Revah

Daniel Vieira Silva

Daniela Aparecida Eufrásio

Daniela da Costa Britto Pereira Lima

Daniela de Oliveira Pires

Daniella Côrtes Pereira Borges

Danilo Cardoso

Danilo Romeu Streck

Danilo Rothberg

Dante Salto

David Poveda Bicknell

Débora Dalbosco Dell'Aglio

Débora Delibertato

Denilson Santos de Azevedo

Denilson Soares Cordeiro
Denise Bianca Maduro Silva

Denise Madeira de Castro e Silva

Denise Meyrelles de Jesus

Denise Trento Rebello de Souza

Denize de Aguiar Xavier Sepulveda

Diana Gonçalves Vidal

Diego Alejandro Pérez

Diego dos Santos Reis

Diego Soares Carvalho

Diléia Aparecida Martins

Dilmeire Sant'Anna

Dilvo Ristoff

Divino José da Silva

Doriedson do Socorro Rodrigues

Douglas Altamiro Consolo

Douglas Christian Ferrari de Melo

Eder Coelho Paula

Edgar Roberto Kirchof

Elaine Meire Vilela

Elenilce Gomes Oliveira

Eliana da Costa Pereira de Menezes

Eliane Feitoza de Oliveira

Eliane Gonçalves

Elias Manuel Said Hung

Elieuza Aparecida de Lima

Élio Carlos Ricardo

Elisabete Ferreira Esteves Campos

Eliseu Raphael Venturi

Elizabeth dos Santos Braga

Emerson Freire

Eneida Simões da Fonseca

Enrique Riquelme Mella

Erika Giacometti Rocha Berribili

Erisson Viana Correa

Eromi Izabel Hummel

Ester Maria Dreher Heuser

Evaldo Piolli

Fabiane Vanessa Breitenbach

Fabiano Ramos Torres

Fábio Franzini

Fábio Hoffmann Pereira

Fabio Vagner Guerino

Fatima Rodríguez Marin

Fernanda Pinto de Aragão Quintino

Fernanda Rodrigues Silva

Fernanda Sarturi

Fernando Luiz Cássio

Fernando Seffner

Filipe Rafael Gracioli

Flávia Inês Schilling

Flavia Medeiros Sarti
Franciane Maria Araldi

Francisca Rodrigues Pini

Gabriela Andrade da Silva

Gabriela Gabriela Medeiros Nogueira

Gabriela Rodella Oliveira

Gabriela Schneider

Geraldo Magela Pereira Leão

Geraldo Romanelli

Gilberto Francisco Dalmolin

Gillka Girardello

Gilson Cruz Junior

Gilvan Müller de Oliveira

Gisela Lobo Baptista Pereira Tartuce

Gisela Maria do Val

Giselle Watanabe

Gladis Massini-Cagliari

Gladys Beatriz Barreyro

Gladys Mary Ghizoni Teive

Graça regina Franco da Silva Reis

Gustavo Henrique Moraes

Helena Montenegro Maggio

Helena Singer

Heloísa Brito de Albuquerque Costa

Heloisa Buarque de Almeida

Heloisa Salles Gentil

Henrique Miguel Lima Silva

Hugo Santos

lara Tatiana Bonin

Ingrid Hotte Ambrogi

Iolanda Alves Évora

Ione Ribeiro Valle

Isabel Carvalho

Isabel de oliveira e silva

Isabel José Fialho

Isabel Melero Bello

Isabela Rosália Lima de Araujo

Ivã Gurgel

Ivani Rodrigues Silva

Iveta Maria Borges Ávila Fernandes

Iza Rodrigues da Luz

Jaciara Sá Carvalho

Jacqueline Moraes Teixeira

Jader Janer Moreira Lopes

Janaina Pamplona da Costa

Jaqueline Dourado do Nascimento

Javier Rodríguez Moreno

Jefferson Galeano Martinez

Jefferson Mainardes

Jeinni Kelly Pereira Puziol

Joana Paulin Romanowski

João do Prado Ferraz de Carvalho 
João Henrique da Silva

Joaquim Cesar Moreira Gama

Joceli de Fatima Arruda Sousa

Joice Sousa Costa

Jonas Marcondes Sarubi de Medeiros

Jonathan Henriques do Amaral

Jorge Osorio Baeza

José Alfredo Oliveira Debortoli

José dos Santos Souza

José Dutra de Oliveira Neto

José Geraldo Silveira Bueno

José Lucas Pedreira Bueno

José Luís Ferraro

José Mauro de Oliveira Braz

José Milton de Lima

José Pedro Cerdeira

Joyce Mary Adam

Juan Carlos Serra

Juan Díaz Godino

Juarez José Tuchinski dos Anjos

Júlia Catani

Juliana de Souza Silva

Juliana França Viol Paulin

Julio César Tovar-Gálvez

Jurema lara Reis Belli

Jussara Cristina Barboza Tortella

Jussara Marques de Macedo

Karina SM Maldonado

Karla Mendonca Menezes

Karla Schuck Saraiva

Kathryn Lum

Katia Arilha Fiorentino Nanci

Kátia Augusta Curado Pinheiro Cordeiro

da Silva

Katia Cristina Silva Forli Bautheney

Katiuscia Cristina Vargas Antunes

Kazumi Munakata

Kelly Cristina Brandão da Silva

Kênia Miranda

Kristie Helene Novoa Durante

Lailson Ferreira Da Silva

Lalo Watanabe Minto

Lara Carlette Thiengo

Lauriana Medeiros Costa Santos

Leandro de Lajonquière

Leidy Gabriela Ariza

Lélia Cristina Silveira de Moraes

Leni Vieira Dornelles

Lenira Haddad

Leonardo Borne

Leonardo Crochik
Leonardo Leite de Andrade

Leonir Lorenzetti

Ligia Paula Couto

Lilian Bacich

Lisete Regina Gomes Arelaro

Livia De Tommasi

Louis José Pacheco de Oliveira

Lucelia Cardoso Cavalcante Rabelo

Lucia Helena Sasseron

Lucia Rabello de Castro

Lúcia Rocha Ferreira

Luciana Backes

Luciana Conrado Martins

Luciana Fernandes Marques

Luciana Garcia de Mello

Luciana Maria Giovanni

Luciana Massi

Luciana Zaterka

Luciano Campos da Silva

Luciano Mendes de Faria Filho

Lucielma de Oliveira Batista Magalhães

de Moura

Lucila Maria Pesce de Oliveira

Lucinéia Rodrigues Magalhães

Luis Eduardo Alvarado Prada

Luis Gustavo D' Carlos Barbosa

Luzia Batista de Oliveira Silva

Lynn Alves

Lynn R. Gama Alves

Maciel Cover

Macioniro Celeste Filho

Maévi Anabel Nono

Magali Aparecida Silvestre

Mani Marins Tebet Azevedo

Manoel Oriosvaldo de Moura

Manuel Alejandro Giovine

Mara Moita

Marcelo Augusto Totti

Marcelo Lima

Marcelo Massa

Márcia Helena de Melo Pereira

Marcia Machado de Lima

Marcia Machado de Lima

Márcia Rodrigues de Souza Mendonça

Márcia Santos da Rocha

Márcia Santos Duarte de Oliveira

Marcílio Souza Júnior

Marcius Cesar Soares Freires

Marco Antonio Coelho Bortoleto

Marco Braga

Marcos Antonio de Almeida
Marcos Antonio Martins Lima

Marcos Francisco Martins

Marcos Garcia Neira

Marcos Namba Beccari

Marcos Sidnei Pagotto Euzebio

Marcus Vinícius Fonseca

Margaret Axt

Margaréte May Berkenbrock Rosito

Maria Amélia Rosário Santoro Franco

Maria Ângela Borges Salvadori

Maria Angélica Pedra Minhoto

Maria Aparecida Bergamaschi

Maria Aparecida de Oliveira Freitas

Maria Carla Corrochano

Maria Cecilia Pérez de Souza-e-Silva

Maria Cristina Cavaleiro

Maria Cristina G. Vicentin

Maria Cristina Menezes

Maria do Carmo de Lacerda Peixoto

Maria do Carmo Meirelles Toledo Cruz

Maria do Socorro Xavier Batista

Maria Eugênia de Lima e Montes Castanho

Maria Fatima Felix Rosar

Maria Flávia Silveira Barbosa

Maria Helena Camara Bastos

Maria Helena da Silva Carneiro

Maria Helena da Silva Ramalho

Maria Helena Santos Gregório

Maria Idalina Krause de Campos

Maria Isabel de Almeida

Maria Isabel Sanchez-Hernandez

Maria José Costa

Maria Leticia Barros Pedroso Nascimento

Maria Lucia Panossian

Maria Lurdes de Carvalho

Maria Nilvane Zanella

Maria Otilia Kroeff Susin

Maria Regina Kawamura

Maria Sueli Correa Dos Prazeres

Marian Ávila de Lima Dias

Mariana de Cássia Assumpção

Mariana Paladino

Mariangela Momo

Marieta Gouvêa de Oliveira Penna

Marilene Dandolini Raupp

Marilene Santos

Marina Pereira de Almeida Mello

Marinez França Souza

Mario Hernández Nodarse

Mario Luiz Neves de Azevedo

Marisa Bittar 
Marisa Gringoletto

Marisa Rossignoli

Maritânia Salete Salvi Rafagnin

Marizabel Kowalski

Marlécio Maknamara

Marlene Guirado

Marli Elisa Dalmazo Afonso de André

Marta Regina Brostolin

Maura Corcini Lopes

Maurício Érnica

Mirian Jorge Warde

Moacyr Aires Novaes Filho

Monica Caldas Ehrenberg

Mônica Cristina Martinez de Moraes

Monica Fantin

Mônica Guimarães Teixeira Amaral

Nadiane Feldkercher

Naiara Silva Ferreira

Nathalya Marillya de Andrade Silva

Núbio Delanne Ferraz Mafra

Odair Sass

Oldimar Pontes Cardoso

Olga Alessandra Mordente

Olga Alicia Gallardo Milanés

Osmar Hélio Alves Araújo

Osvaldo Luiz Ferraz

Pablo Beltrán-Pellicer

Pâmela Félix Freitas

Patricia Cristina Albieri de Almeida

Patrícia da Silva Sessa

Patrícia Simone Nogueira

Patrício Carneiro Araújo

Patricio Lepe-Carrión

Paulo Alberto dos Santos Vieira

Paulo Brazão

Paulo Henrique Fernandes Silveira

Paulo Roberto Menezes Lima JR

Paulo Rogério da Conceição Neves

Paulo Vitor de souza Pinto

Pedro Angelo Pagni

Pedro Jorge da Costa Caetano

Poliana Bruno Zuin

Preciosa Fernandes

Priscila Monteiro Chaves

Priscila Moreira Corrêa Telles

Rafael Petta Daud
Rafaela Marques

Rafaele Rodrigues de Araújo

Raphael de Andrade Ribeiro

Raquel de Oliveira Mendes

Raquel Milani

Regina Cândida Ellero Gualtieri

Regina Tereza Cestari de Oliveira

Régis Henrique dos Reis Silva

Renata Lopes Costa Prado

Renata P Lima Aspis

Renato Marcone José de Souza

Ricardo Boklis Golbspan

Ricardo Desidério Da Silva

Rita de Cássia Marchi

Rita Marisa Ribes Pereira

Rita Santos Guimarães

Roberto da Silva

Roberto Francisco de Carvalho

Romilda Teodora Ens

Roni Cleber Dias de Menezes

Rosa Fátima de Souza Chaloba

Rosa Maria Bueno Fischer

Rosa Maria Hessel Silveira

Rosana Louro Ferreira Silva

Rosângela Gavioli Prieto

Rosario Gil-Galvan

Rosário Silvana Genta Lugli

Roseli Rodrigues de Mello

Rosenilton da Silva Oliveira

Rubens Barbosa de Camargo

Russel Teresinha Dutra da Rosa

Sandra Maria Sawaya

Sandra Regina Buttros Gattolin

Sandra Valenzuela

Sandra Zákia Sousa

Savio Gonçalves dos Santos

Selma Garrido Pimenta

Sergio Antonio Carlos

Sergio Paulino Abranches

Sidney Jard da Silva

Silvia Christina de Oliveira Madrid

Silvia Lucia Bigonjal Braggio

Sílvia Maria Agatti Lüdorf

Silvio Donizetti de Oliveira Gallo

Sílvio Luiz de Paula

Silvio Ricardo Gomes Carneiro
Simone Braz Ferreira Gontijo

Simone Eliza do Carmo Lessa

Sinara Pollom Zardo

Sirlene Aparecida Aarão

Sonia Maria Portella Kruppa

Sônia Regina dos Santos Teixeira

Sônia Teresinha Sousa Penin

Soraya Cristina Pacheco de Meneses

Stela Mithá Duarte

Sueli Lima Moreira

Sueli Soares dos Santos Batista

Suely dos Santos Silva

Sumaya Mattar

Suzana dos Santos Gomes

Taís Cristine Ernst Frizzo

Tatiana Luiza Rech

Tel Amiel

Teresa Cristina Rego

Theresa Adrião

Thiago dos Santos Molina

Túlio Campos

Ulisses Ferreira de Araujo

Valdemar Sguissardi

Valdeniza da Barra

Valdir Heitor Barzotto

Valeria Llobet

Valmir Flôres Pinto

Vanda Mendes Ribeiro

Vanessa Ferraz Almeida Neves

Vanessa Teixeira Pipinis

Vera Lucia Gaspar da Silva

Vera Teresa Valdemarin

Verilda Speridião Kluth

Verónica Marcela Guridi

Vinicius Cavalcanti Ferreira

Vivian Batista da Silva

Viviane Castro Camozzato

Viviane Potenza Guimarães Pinheiro

Viviane Toraci Alonso de Andrade

Wagner Teixeira Dias

Wania Regina Coutinho Gonzalez

Wilma Favorito

Wilma Pastor de Andrade Sousa

Wilson Mesquita Almeida

Ygor Corrêa

Zaqueu Vieira Oliveira 